\title{
Influencia de la ansiedad en el aprendizaje del idioma inglés como lengua extranjera en estudiantes de educación superior
}

\section{Anxiety influence on learning English as a foreign language in higher education students}

\author{
Cyntia Sofía Adrianzén Segovia \\ Universidad Científica del Sur, Lima, Perú \\ cyntiasofia@gmail.com \\ https://orcid.org/0000-0002-4849-7066
}

Recepción: 14/04/2021 | Aceptación: 31/05/2021 | Publicación: 10/09/2021

Cómo citar (APA, séptima edición):

Adrianzén Segovia, C. S. (2021). Influencia de la ansiedad en el aprendizaje del idioma inglés como lengua extranjera en estudiantes de educación superior. INNOVA Research Journal, 6(3), 58-78. https://doi.org/10.33890/innova.v6.n3.2021.1742

\section{Resumen}

La presente investigación tuvo como objetivo determinar el nivel de ansiedad frente al aprendizaje de una lengua extranjera, como el caso del idioma inglés, por lo que se consideró como referencia a 37 estudiantes de un instituto de educación superior de Lima, Perú. La muestra la conformaron mujeres y varones. Los instrumentos utilizados fueron: The Foreign Language Classroom Anxiety Scale (FLCAS) para evaluar la ansiedad y un instrumento para medir la habilidad speaking como parte del aprendizaje del idioma inglés. Se observó un nivel de ansiedad medio y el principal componente fue la ansiedad ante los exámenes.

Palabras claves: educación bilingüe; ansiedad en el aprendizaje.

\begin{abstract}
The objective of this research was to determine the level of anxiety in the face of learning a foreign language, in this case the English language, for which 37 students from a higher education institute in Lima, Peru were considered as a reference. The sample was made up of women and men. The instruments used were: The Foreign Language Classroom Anxiety Scale (FLCAS) to assess anxiety and an instrument to measure speaking ability as part of learning the English language. A medium level of anxiety was observed and the main component was test anxiety.
\end{abstract}

Keywords: bilingual education; learning anxiety. 


\section{Introducción}

El aprendizaje de una lengua extranjera se ha convertido en una necesidad en el mundo actual debido a que con su conocimiento accedemos a mejores oportunidades de estudio y trabajo lo que nos permite optimizar nuestra calidad de vida. Nos encontramos inmersos en un mundo globalizado que nos exige a ser cada vez más eficientes en los ámbitos personal y profesional con el objetivo de tener una formación integral para poder hacer frente a las necesidades que demanda el mercado laboral actual.

En este contexto, la importancia de contar con el dominio de una lengua extranjera es fundamental ya que nos permite estar al día de los constantes cambios que surgen a nivel científico, tecnológico, comercial y financiero en el mundo donde el idioma inglés ocupa un lugar preponderante (aproximadamente 1, 5000 millones de personas en el mundo hablan inglés, de las cuales solo 375 millones son hablantes nativos) ${ }^{1}$. Además, es la lengua oficial no solo de varios países sino también de organismos internacionales como la Organización de las Naciones Unidas (ONU), entre otras entidades.

En el caso de nuestro país, el aprendizaje del idioma inglés es una parte esencial del currículo tanto en las escuelas públicas y privadas además de otras instituciones educativas como las universidades e institutos de educación superior donde como parte de los requisitos para obtener el grado académico correspondiente es necesario contar con el conocimiento de un idioma extranjero tal como lo indica los lineamientos académicos generales (2018) en el punto 14.3.1

Para la obtención del grado de bachiller técnico, se requiere acreditar el idioma extranjero, de preferencia el inglés en el nivel B1 y para la obtención del grado de bachiller, se requiere acreditar el idioma extranjero, de preferencia el inglés en el nivel B2, de acuerdo a los parámetros del Marco Común Europeo de Referencias para las Lenguas Modernas al que hace referencia la Política Nacional de Enseñanza, Aprendizaje y Uso del idioma inglés, Puertas al Mundo, aprobado por Decreto Supremo Nº12-2015-MINEDU.

Es por estas razones que el idioma inglés forma parte esencial en el desarrollo de nuestros estudiantes ya que no solo aprenden un nuevo idioma sino también acceden al conocimiento de una nueva cultura. La instrucción del inglés como lengua extranjera a nivel superior está enfocada en función de la participación activa del estudiante dentro de su propio aprendizaje a través del uso de fuentes primarias que les permita la interacción significativa entre sus conocimientos y el contenido impartido. (Guanipa, 2001; Govea y Sánchez, 2006; Pereira y Ramírez, 2008).

En lo que se refiere al proceso de aprendizaje de toda lengua extranjera se han investigado una serie de variables entre las que más resaltan las cognitivas como la inteligencia y la aptitud; las variables afectivas como la motivación y la ansiedad y las variables individuales entre las que se encuentran el género, la edad, entre otras. Los estudios ejecutados a la variable afectiva de la ansiedad en relación con el aprendizaje de lenguas extranjeras se han realizado con el objetivo de conocer principalmente el nivel de interferencia que esta puede tener en la persona afectando dicho aprendizaje.

\footnotetext{
${ }^{1}$ Fuente: los idiomas más hablados en el mundo a fecha 2020
} 
Por lo mencionado anteriormente, esta investigación se plantea la siguiente interrogante: ¿Cuál es la relación entre la ansiedad y el aprendizaje del idioma inglés como lengua extranjera en estudiantes de un instituto de educación superior de Lima? Además, el objetivo general es determinar el nivel de ansiedad en el aprendizaje del idioma inglés como lengua extranjera en estudiantes de un instituto de educación superior de Lima. Propone como objetivos específicos determinar la relación de cada una de estos tres componentes: la aprehensión comunicativa, el temor a la evaluación negativa de parte de los compañeros; y la ansiedad frente a los exámenes con el aprendizaje del idioma inglés como lengua extranjera en estudiantes de un institu to de educación superior de Lima. Los resultados de esta investigación van a mejorar el conocimiento que debemos tener como docentes sobre las necesidades de nuestros estudiantes con el objetivo de contribuir a aminorar la sensación de ansiedad al momento de aprender una lengua extranjera y mejorar su desempeño.

\section{Revisión Literaria}

En el transcurso de los años se han realizado diversas definiciones sobre qué es la ansiedad según los síntomas físicos y emocionales que provoca y dependiendo del contexto en el que se da. Desde el punto de vista psicológico, la ansiedad es una respuesta emocional que se presenta en la persona ante situaciones que percibe como amenazantes o peligrosas resultando perjudicial ya que se vuelve frecuente (Virues, 2005).

Según el Manual Diagnóstico y Estadístico de los trastornos mentales (DSM-IV-TR) la ansiedad generalizada se caracteriza por una ansiedad y preocupación en exceso sobre una variedad de acontecimientos durante más de seis meses que afectan la vida de la persona presentándose síntomas como inquietud, irritabilidad, dificultad para concentrase por mencionar algunos.

Para MacIntyre y Gardner (1991) existen tres categorías en relación a la ansiedad: La ansiedad de rasgo, se refiere al estado emocional relativamente estable que una persona experimenta con más frecuencia o más intensidad que la mayoría de las personas en promedio y es parte de su propia personalidad. La ansiedad estado, se refiere a la respuesta que se da ante un estímulo concreto. Según, MacIntyre (1999) puede ser causada por rendir un examen, hablar en público, conocer a los padres de la pareja o intentar hablar en otro idioma. La ansiedad ante situaciones específicas, se define como la probabilidad de sentirse ansioso ante una situación particular como cuando se está dando un examen, solucionando un problema de matemáticas o hablando en un segundo idioma. Para Horwitz (2001) el aprendizaje de lenguas extranjeras es un tipo de ansiedad específica ya que se da dentro del aula en el contexto de dicho aprendizaje.

En lo que concierne a la educación a nivel superior, Bertoglia (2015) señala que la ansiedad es propia de estudiantes de educación superior ya que afrontan situaciones estresantes producto del contexto en el cual se encuentran inmersos y no son capaces de canalizar o exteriorizar de manea asertiva la ansiedad produciendo consecuencias nefastas en su salud mental.

En términos generales, la ansiedad es un estado de agitación e inquietud desagradable que se caracteriza por la anticipación del peligro, el predominio de síntomas psíquicos y la sensación de catástrofe o de peligro inminente. Todas las personas en algún momento de nuestra vida hemos 
Influencia de la ansiedad en el aprendizaje del idioma inglés como lengua extranjera en estudiantes de educación superior

experimentado cierto grado de ansiedad, pero no del mismo modo ni en las mismas circunstancias. Sin embargo, es necesario prestarle atención cuando se presenta de manera constante ya que afectaría no solo física y psicológicamente la actividad de una persona. Ya descrito algunos conceptos sobre la ansiedad en diversas dimensiones pasaremos a revisar de manera específica los estudios relacionados al presente tema de investigación.

\section{La ansiedad en el aprendizaje de lenguas extranjeras}

En el transcurso de los años se han realizado varios estudios con el objetivo de conocer el nivel de ansiedad que una persona puede desarrollar en relación al aprendizaje de una lengua extranjera ya que afecta de manera efectiva o ineficiente el mismo. Debido a que varios autores han definido la ansiedad en el aprendizaje del idioma extranjero, nos centraremos en los más relevantes para el presente estudio.

Para MacIntyre \& Gardner (1994) la ansiedad en el aprendizaje de la lengua extranjera es un sentimiento de tensión y aprensión asociado específicamente a contextos que se dan en la adquisición de segundas lenguas incluyendo la producción oral, la comprensión oral y el aprendizaje. Resulta complejo el estudio de la ansiedad y las lenguas extrajeras debido a una combinación de diversos factores y contextos en la que se da.

Posteriormente, MacIntyre (1999) señala que este tipo de ansiedad puede tener una serie de efectos en lo académico, cognitivo, social y personal. En lo académico, como se menciona más adelante en los estudios de Horwitz (1986) y Aida (1994) donde se muestra niveles altos de ansiedad en el aprendizaje de la lengua extranjera asociados a niveles bajos de rendimiento académico en el curso de idiomas. En lo cognitivo, se da en tres etapas: la de entrada, procesamiento y salida de la información. En lo social, se trata de la disponibilidad que tienen los estudiantes para comunicarse durante la clase donde se suele comparar entre ellos esta habilidad. En lo personal, se plantea cómo cada persona afronta la ansiedad en este contexto ya que para algunos el aprendizaje de la lengua extranjera puede ser una experiencia poco agradable.

Sin embargo, el estudio que más interés ha suscitado es el de Horwitz et al. (1986) sobre el concepto de ansiedad vinculado al aprendizaje de lengua extranjeras en un contexto o situación específica refiriéndose a la misma como "FLA is a distinct complex construct of self-perceptions, beliefs, feelings, and behaviours related to classroom language learning arising from the uniqueness of language learning process" (p. 128). Además, son los creadores de "Foreign language class anxiety scale" (FLCAS) una escala para medir el nivel de ansiedad linguística de los estudiantes en el proceso de aprendizaje de una lengua extranjera utilizada en varios estudios debido a su alto grado de confiabilidad y el que será utilizado en la presente investigación. Estos autores estudiaron por primera vez los componentes de la ansiedad en dicho aprendizaje produciendo un efecto negativo en el mismo:

- La aprensión comunicativa: se asocia a la timidez del estudiante en contextos donde tiene que comunicarse. Es decir, el estudiante se siente frustrado al no entender a otra persona o cuando no se puede comunicar de manera eficiente. Para Horwitz et al. existen tres situaciones que desarrollan este componente: hablar en público, hablar con otra persona o hablar en un grupo escuchando un mensaje hablado.

Esta obra se comparte bajo la licencia Creative Common Atribución-No Comercial 4.0 International (CC BY-NC 4.0) Revista de la Universidad Internacional del Ecuador. URL: https://www.uide.edu.ec/ 
- El temor a la evaluación negativa por parte de los compañeros: se relaciona a las percepciones que los estudiantes piensan que sus demás compañeros tienen sobre ellos. Este componente puede afectar de manera negativa al estudiante tanto en el aspecto cognitivo como el emocional. En el primer caso al dificultar su capacidad para prestar atención y comprender nueva información; y en el segundo al generar sentimientos como la ansiedad.

- La ansiedad frente a los exámenes: se trata de las expectativas de los estudiantes por obtener altas notas y tener éxito en la clase de lengua extranjera en la que constantemente se realizan una serie de exámenes y evaluaciones con el objetivo de comprobar el avance de los estudiantes. Este proceso puede elevar los niveles de estrés y presión en los estudiantes que sienten más ansiedad.

Según Criado \& Mengual (2017) La importancia de entender estos tres componentes proviene de los diversos efectos que se dan en las diferentes variables que afectan la adquisición de lenguas extranjeras como la auto-percepción, el éxito, etc. Además, esta escala ha sido utilizada en diversos estudios como el de Alcántara (1992) que la utilizo para medir el nivel de ansiedad en dos grupos que estudiaban inglés. Uno de ellos utilizaba el método suggestopedia y el otro utilizaba un método tradicional encontrando que no hay diferencias significativas entre estos dos grupos.

Más adelante, en un estudio hecho por Aida (1994) se mostraron resultados similares al estudio de Horwitz en el que se realizó un análisis factorial del FLCAS a 96 estudiantes universitarios que estudiaban japonés con el objetivo de encontrar los dos tipos de ansiedad que Horwitz et al. (1986) mencionaban presentarse en el aprendizaje de lenguas extranjeras donde los componentes principales hallados fueron el temor al fracaso y la ansiedad al hablar en la lengua extranjera.

Posteriormente, Ortega Cebreros (2003) utilizo la versión del FLCAS adaptado al español y a sus intereses. El instrumento fue aplicado a estudiantes universitarios encontrándose niveles altos de ansiedad en las clases de lengua extranjera ya que un 69\% de los estudiantes sintió temor de equivocarse al hablar en inglés, un 57\% no se sintieron cómodos al no entender lo que el profesor corregía y finalmente, un $72 \%$ de los estudiantes expresaron no sentirse a gusto durante los exámenes de lengua extranjera. Esto se relaciona con que el $88 \%$ de los estudiantes estaban preocupados por las consecuencias de no aprobar dicha asignatura.

Es necesario también mencionar la hipótesis del Filtro Afectivo hecha por Krashen (1987) donde se señala a los factores emocionales (la motivación, la ansiedad, la autoestima, entre otros) como elementos que se relacionan de manera directa al proceso de aprendizaje de una segunda lengua y que afectan a los estudiantes de una u otra manera.

Por otro lado, existen diferentes investigaciones concentradas en la relación de la ansiedad con las habilidades que se desarrollan como parte del aprendizaje de una lengua extranjera tales como la comprensión escrita y oral (reading \& listening); producción escrita y oral (writing \& speaking). Nos centraremos en los estudios relacionados a la producción oral (speaking) ya que es una de las habilidades que genera niveles de ansiedad más altos dentro del aprendizaje de lenguas extranjeras tal como lo señalo Bekleyen (2004) y se desea evidenciar en el presente trabajo. Así tenemos que Young (1999) investigó las percepciones de los estudiantes en relación a la ansiedad 
Influencia de la ansiedad en el aprendizaje del idioma inglés como lengua extranjera en estudiantes de educación superior

y al momento de hablar durante la clase de idiomas dando como resultado que los estudiantes sentían niveles altos de ansiedad al momento de comunicarse frente a sus compañeros.

\section{Metodología}

Para Fernández, Hernández y Baptista (2004) definen el diseño de investigación como la estrategia o plan preparado con el objetivo de explicar las inquietudes en el proceso de investigación y lograr sus objetivos de modo concreto y práctico. Este estudio es de tipo no experimental, correlacional y transeccional con enfoque cuantitativo debido a que es una muestra no probabilística intencional ya que la misma es representativa para el trabajo llevado a cabo.

A continuación, se procederá a realizar la investigación de campo con el objetivo de ratificar nuestra hipótesis general: Existe influencia significativa de la ansiedad en el aprendizaje del idioma inglés como lengua extranjera en estudiantes de un instituto de educación superior de Lima. Dado que la ansiedad y los procesos cognitivos están relacionados de manera significativa, si afectaría si se presenta de manera alta y moderada entre los estudiantes. De presentarse este contexto, procesos como la atención, la memoria, la toma de decisiones, la solución de problemas, entre otros podrían verse afectados. En un estudio realizado por Cano (1997) indica que tanto la ansiedad como el estrés disminuyen el rendimiento en tareas dificultosas por lo que se necesita un nivel medio u óptimo de ansiedad para lograr un alto rendimiento. Es por esta razón que se desea verificar esta hipótesis además de las siguientes hipótesis específicas:

Existe influencia significativa de la aprehensión comunicativa en el aprendizaje del idioma inglés como lengua extranjera en estudiantes de un instituto de educación superior de Lima.

Existe influencia significativa del temor a la evaluación negativa por parte de los compañeros en el aprendizaje del idioma inglés como lengua extranjera en estudiantes de un instituto de educación superior de Lima.

Existe influencia significativa de la ansiedad frente a los exámenes en el aprendizaje del idioma inglés como lengua extranjera en estudiantes de un instituto de educación superior de Lima.

\section{Participantes}

Los participantes que han colaborado con la investigación son estudiantes de una institución de educación superior de Lima. El aprendizaje del idioma inglés forma parte de la malla curricular de la institución por tal motivo es obligatorio que los estudiantes participen de este curso. El número de participantes es 37,28 varones $(75,7 \%)$ y 9 mujeres $(24,3 \%)$ cuyas edades comprenden entre los 18 y 25 años. Estos datos se observan en la figura 1. 


\section{Figura 1}

Muestra por género

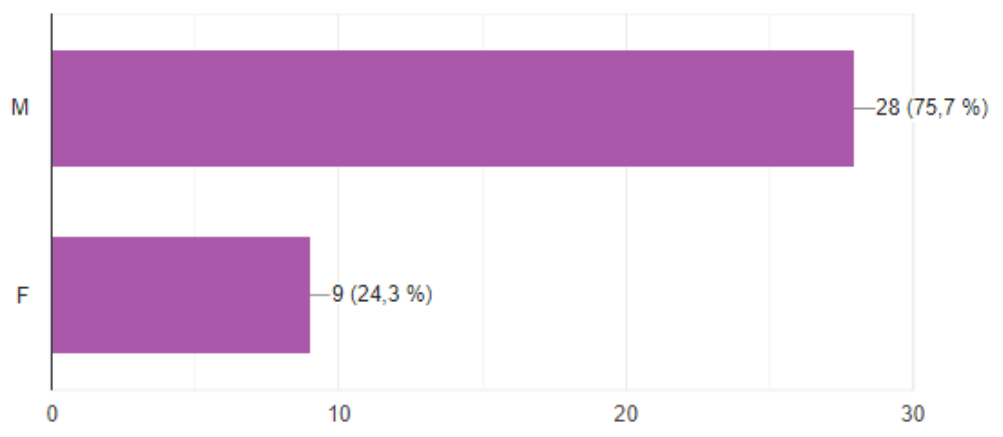

\section{Instrumentos}

La presente investigación utilizó dos instrumentos para obtener los datos. En primer lugar, se utilizó "Foreign Language Anxiety Scale" diseñado por Horwitz et al. (1986). traducido al español por Pérez-Paredes y Martínez-Sánchez (2000-2001). El cuestionario ha sido adaptado nuevamente para poder aplicarlo con nuestros estudiantes. Al realizar esta prueba se desea conocer la presencia de la ansiedad en el aprendizaje de la lengua extranjera, en este caso el idioma inglés. Este cuestionario está compuesto por 33 ítems que se miden con la escala de Likert de cinco puntos donde las respuestas se expresan de menor a mayor grado de conformidad donde las respuestas para cada afirmación son: totalmente en desacuerdo, en desacuerdo, no sé, de acuerdo y totalmente de acuerdo. Al comienzo del cuestionario se incluyen dos preguntas para completar: edad y sexo.

En segundo lugar, se utilizó un cuestionario basado en las rubricas dadas por la institución para obtener información específica en cuanto la expresión oral (speaking) ya que, como se menciona anteriormente, genera niveles altos de ansiedad en el aprendizaje del idioma inglés. Este cuestionario está compuesto por 6 ítems que también se miden con la escala de Likert de cuatro puntos donde las respuestas igualmente se expresan de menor a mayor grado de conformidad para cada afirmación: nunca, a veces, casi siempre y siempre. Se realizó la validación de los dos instrumentos a través del juicio de expertos para brindar mayor respaldo a la investigación.

\section{Fiabilidad de los instrumentos}

Para determinar la confiabilidad se realizó una prueba piloto a 10 estudiantes con similares características de la muestra original de estudio con el propósito de evaluar el comportamiento de dichos instrumentos en el momento de la toma de datos para la consistencia interna. Los datos se procesaron a través de la prueba de confiabilidad de alfa de Cronbach para estimar la consistencia interna del instrumento, cuya fórmula es:

$$
\alpha=\frac{K}{K-1}\left[1-\frac{\sum S_{i}^{2}}{S_{T}^{2}}\right]
$$


Donde:

K: número de preguntas o ítems.

Si2: sumatoria de varianza de los ítems.

ST 2: varianza de la suma de los ítems.

$\alpha$ : alfa de Cronbach

\section{Tabla 1}

Análisis de fiabilidad del instrumento que mide el nivel de ansiedad

\begin{tabular}{lc}
\hline Alfa de Cronbach & N de elementos \\
\hline 0,885 & 33 \\
\hline \multicolumn{2}{c}{ Habría alta consistencia interna de la escala }
\end{tabular}

Habría alta consistencia interna de la escala

\section{Tabla 2}

Análisis de fiabilidad del instrumento que mide el aprendizaje

\begin{tabular}{cc}
\hline Alfa de Cronbach & N de elementos \\
\hline 0,926 & 6 \\
\hline \multicolumn{2}{c}{ Habría alta consistencia interna de la escala }
\end{tabular}

\section{Recogida de datos}

Se solicitó la autorización al departamento académico para la aplicación de los cuestionarios que se pasaron en el mes de noviembre a través de una encuesta online (Google forms) y los estudiantes dispusieron de 30 minutos para completarlos. Previamente, se explicó de qué tratan y se indicó que ante alguna dificultad durante el llenado de los cuestionarios no duden en preguntar y se reiteró el anonimato de los mismos además de la nula repercusión en sus notas de la asignatura de inglés.

\section{Análisis de datos}

Los resultados de la investigación se han divido de la siguiente manera: la aprensión comunicativa, el temor a la evaluación negativa y la ansiedad frente a los exámenes. Es decir, los componentes de la ansiedad en lo referente al primer cuestionario. El segundo cuestionario queda divido de la siguiente manera: vocabulario y gramática, pronunciación y entonación; contenido y límite de tiempo en lo referente a la producción oral dentro del aprendizaje del idioma inglés. 


\section{Resultados}

El análisis descriptivo correspondiente a las variables de estudio: Ansiedad y Aprendizaje del idioma inglés con sus respectivas componentes; arrojan los resultados que se muestran en las siguientes tablas con los niveles de medición correspondiente por cada variable.

\section{Escala valorativa de la variable ansiedad de los estudiantes}

En la tabla siguiente se detallan los niveles, rangos y los puntajes teóricos, donde la variable ansiedad de los estudiantes alcanza 170 puntos, en el caso de los componentes, los puntajes teóricos se encuentran entre 7 y 78 puntos.

\section{Tabla 3}

Escala valorativa de la variable ansiedad de los estudiantes

\begin{tabular}{llllll}
\hline \multirow{2}{*}{ Variable y componentes } & \multicolumn{3}{c}{ Puntajes } & \multicolumn{3}{c}{ Niveles } \\
\cline { 2 - 6 } & Mínimo & Máximo & Bajo & Medio & Alto \\
\hline Ansiedad del estudiante & 63 & 170 & $63-98$ & $99-134$ & $135-170$ \\
Aprehensión comunicativa & 18 & 47 & $18-27$ & $28-37$ & $38-47$ \\
Temor a la evaluación negativa por & 11 & 78 & $11-32$ & $33-54$ & $55-78$ \\
parte de los compañeros & 32 & 55 & $32-39$ & $40-47$ & $48-55$ \\
Ansiedad frente a los exámenes & 32 & & & & \\
\hline
\end{tabular}

\section{Escala valorativa de la variable aprendizaje del idioma inglés}

En la siguiente tabla se detallan los niveles, rangos y los puntajes teóricos, donde la variable aprendizaje del idioma inglés alcanza 18 puntos, en el caso de los componentes, los puntajes teóricos se encuentran entre 2 y 8 puntos.

\section{Tabla 4}

Escala valorativa de la variable aprendizaje del idioma inglés

\begin{tabular}{llllll}
\hline \multirow{2}{*}{ Variable y componentes } & \multicolumn{3}{c}{ Puntajes } & \multicolumn{3}{c}{ Niveles } \\
\cline { 2 - 6 } & Mínimo & Máximo & Bajo & Medio & \multicolumn{1}{c}{ Alto } \\
\hline Aprendizaje del idioma inglés & 7 & 18 & $7-10$ & $11-14$ & $15-18$ \\
Vocabulario y gramática & 2 & 6 & $2-3$ & $4-5$ & 6 \\
Pronunciación y entonación & 2 & 8 & $2-3$ & $4-5$ & $6-8$ \\
Contenido y límite de tiempo & 2 & 6 & $2-3$ & $4-5$ & 6 \\
\hline
\end{tabular}


Influencia de la ansiedad en el aprendizaje del idioma inglés como lengua extranjera en estudiantes de educación superior

Análisis descriptivo de los resultados de la variable ansiedad de los estudiantes y sus componentes

\section{Tabla 5}

Niveles de distribución de los componentes de la ansiedad de los estudiantes

\begin{tabular}{|c|c|c|c|c|c|c|c|c|}
\hline & \multicolumn{2}{|c|}{ Bajo } & \multicolumn{2}{|c|}{ Medio } & \multicolumn{2}{|c|}{ Alto } & \multicolumn{2}{|r|}{ Total } \\
\hline & $\mathbf{n}$ & $\%$ & $\mathbf{n}$ & $\%$ & $\mathbf{n}$ & $\%$ & $\mathbf{n}$ & $\%$ \\
\hline Aprehensión comunicativa & 33 & $89.2 \%$ & 3 & $8.1 \%$ & 1 & $2.7 \%$ & 37 & $100 . \%$ \\
\hline $\begin{array}{l}\text { Temor a la evaluación } \\
\text { negativa por parte de los } \\
\text { compañeros }\end{array}$ & 22 & $59.5 \%$ & 11 & $29.7 \%$ & 4 & $10.8 \%$ & 37 & $100 . \%$ \\
\hline $\begin{array}{l}\text { Ansiedad frente a los } \\
\text { exámenes }\end{array}$ & 13 & $35.1 \%$ & 11 & $29.7 \%$ & 13 & $35.1 \%$ & 37 & $100 . \%$ \\
\hline
\end{tabular}

\section{Figura 2}

Niveles de distribución de las componentes de la ansiedad de los estudiantes

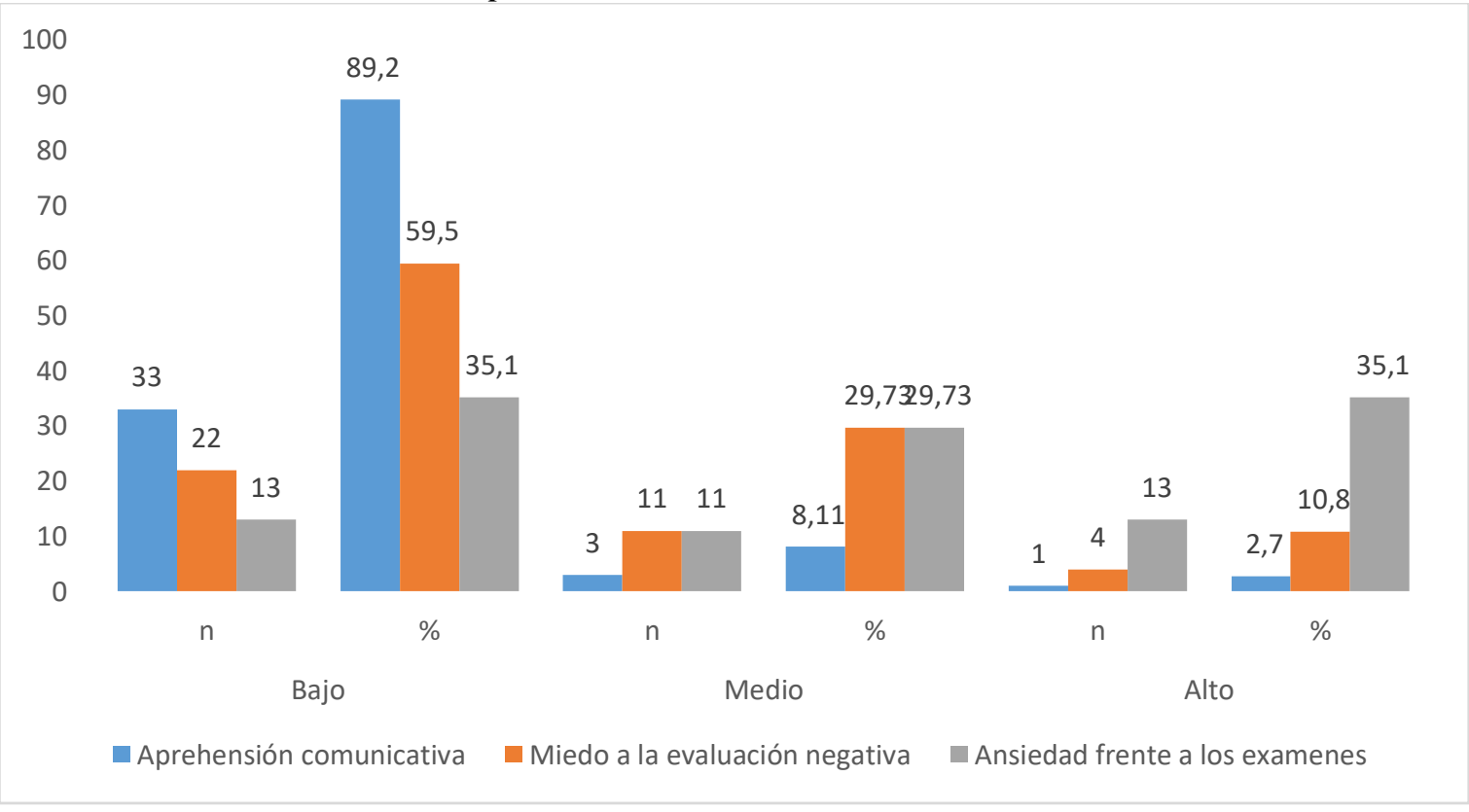

En la tabla 5 y figura 2, referente a los componentes de la ansiedad de los estudiantes, se evidencia que del total de 37 estudiantes de inglés de un instituto de educación superior de Lima, 33 estudiantes que representan el 89.2\% muestran un bajo nivel en el componente aprehensión 
comunicativa, el $8.11 \%$ (3) muestran un nivel medio de ansiedad; mientras que $2.7 \%$ (1) muestran un alto nivel en la componente aprehensión comunicativa.

En cuanto al segundo componente referente al temor de los estudiantes a la evaluación negativa por parte de los compañeros, $59.5 \%$ (22) muestran un bajo nivel; $29.73 \%$ (11) evidencian un nivel medio y el $10.8 \%$ (4) muestran un alto nivel.

En el tercer componente referente a la ansiedad de los estudiantes frente a los examenes, $35.1 \%$ (13) muestran un bajo nivel; $29.73 \%$ (11) muestran un nivel medio; mientras que $35.1 \%$ (13) se encuentran con un alto nivel.

\section{Tabla 6}

Niveles de distribución de la ansiedad de los estudiantes

\begin{tabular}{llll}
\hline & & Frecuencia & Porcentaje \\
\hline \multirow{4}{*}{ Nivel } & Bajo & 17 & 45,9 \\
& Medio & 18 & 48,6 \\
& Alto & 2 & 5,4 \\
& Total & 37 & 100,0 \\
\hline
\end{tabular}

\section{Figura 3}

Distribución de las frecuencias de la variable ansiedad de los estudiantes

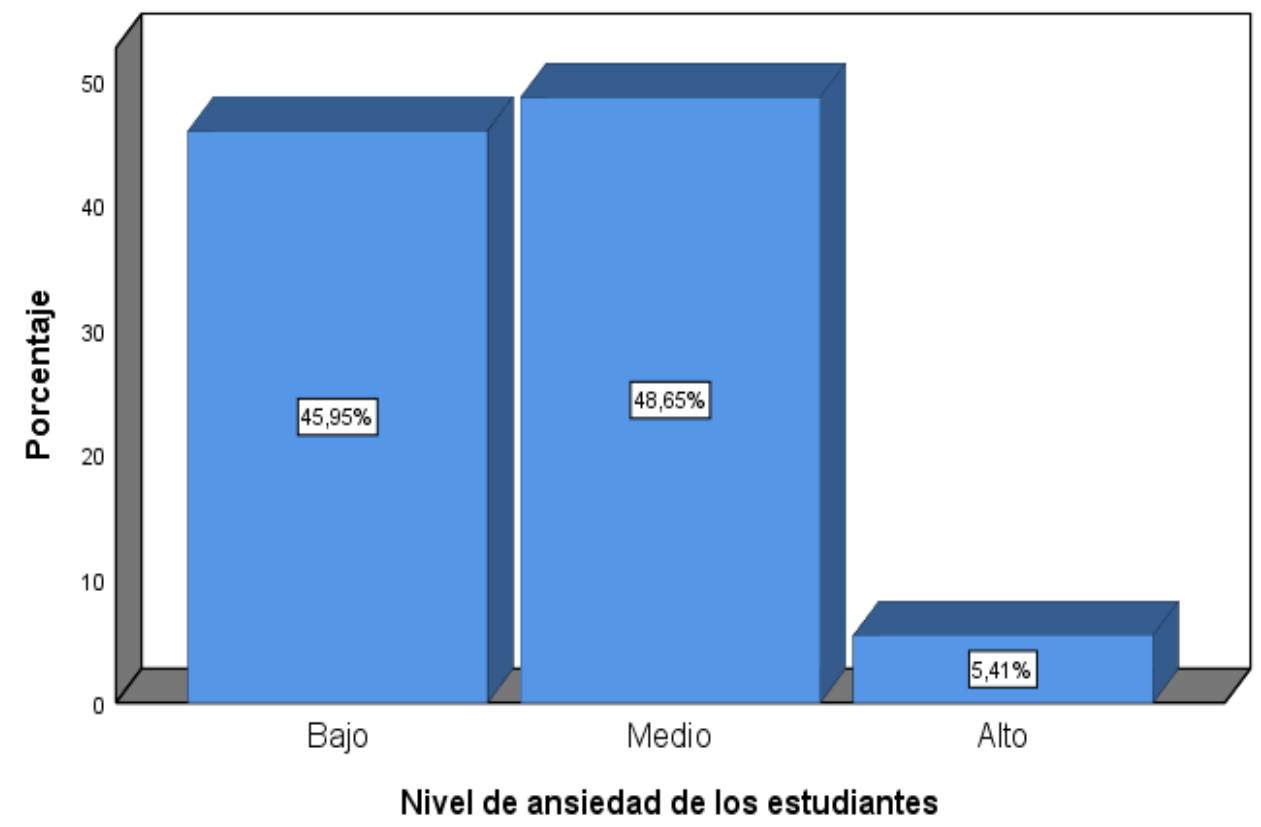


Influencia de la ansiedad en el aprendizaje del idioma inglés como lengua extranjera en estudiantes de educación superior

En la tabla 6 y figura 3 referente a la ansiedad de los estudiantes de un instituto de educación superior, observamos que $45.9 \%$ (17) se encuentran con un bajo nivel de ansiedad frente al aprendizaje de inglés; $48.6 \%$ (18) evidencian un nivel medio; mientras que 5.4\% (2) muestran un alto nivel.

\section{Tabla 7}

Niveles de distribución de los componentes del aprendizaje del idioma inglés

\begin{tabular}{lcccccccc}
\hline & \multicolumn{2}{c}{ Bajo } & \multicolumn{2}{c}{ Medio } & \multicolumn{2}{c}{ Alto } & \multicolumn{2}{c}{ Total } \\
\cline { 2 - 9 } & $\mathbf{n}$ & $\mathbf{\%}$ & $\mathbf{n}$ & $\mathbf{\%}$ & $\mathbf{n}$ & $\mathbf{\%}$ & $\mathbf{n}$ & \% \\
\hline Vocabulario y gramática & 23 & 62.2 & 11 & 29.7 & 3 & 8.1 & 37 & 100 \\
Pronunciación y entonación & 12 & 32.4 & 20 & 54.1 & 5 & 13.5 & 37 & 100 \\
Contenido y límite de tiempo & 18 & 48.6 & 14 & 37.8 & 5 & 13.5 & 37 & 100 \\
\hline
\end{tabular}

\section{Figura 4}

Niveles de distribución de las componentes del aprendizaje del idioma inglés

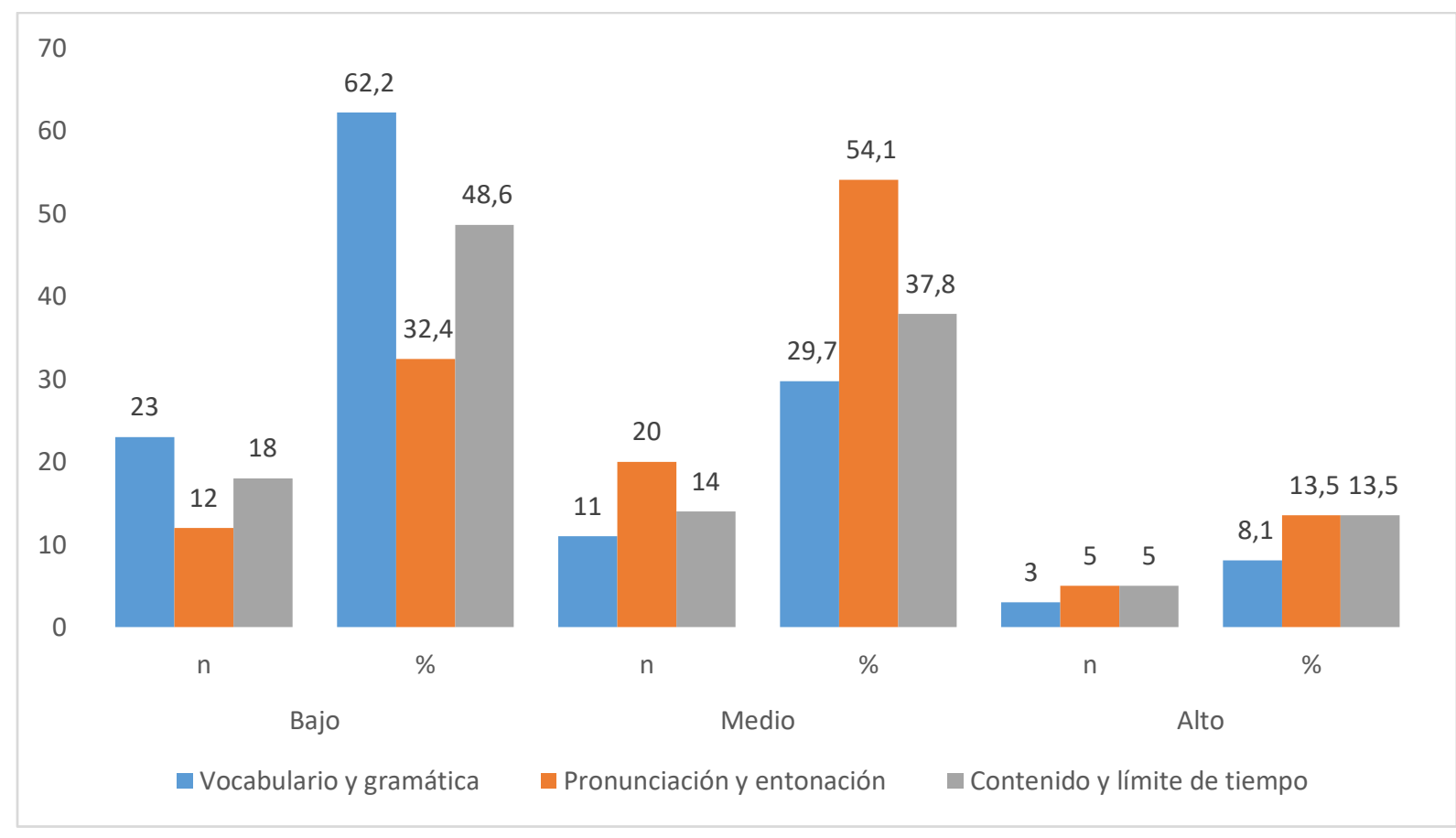

En la tabla 7 y figura 4, referente a los componentes del aprendizaje del idioma inglés, se evidencia que del total de 37 estudiantes de inglés de un instituto de educación superior de Lima, 23 estudiantes que representan el $62.2 \%$ muestran un bajo nivel en en cuanto al vocabulario y 
gramática, el 29.7\% (11) muestran un nivel medio; mientras que 8.1\% (3) muestran un alto nivel en cuanto al vocabulario y gramática.

En cuanto al segundo componente referente a la pronunciación y entonación de los estudiantes de inglés de un instituto de educación superior de lima, 32.4\% (12) muestran un bajo nivel; $54.1 \%$ (20) evidencian un nivel medio y el $13.5 \%$ (5) muestran un alto nivel. En el tercer componente referente al contenido y límite de tiempo de los estudiantes de inglés de un instituto superior de Lima, 48.6\% (18) muestran un bajo nivel; 37.8\% (14) muestran un nivel medio; mientras que $13.5 \%$ (5) se encuentran con un alto nivel en este componente.

\section{Tabla 8}

Niveles de distribución del aprendizaje del idioma inglés

\begin{tabular}{llll}
\hline & & Frecuencia & Porcentaje \\
\hline \multirow{3}{*}{ Nivel } & Bajo & 12 & 32,4 \\
& Medio & 12 & 32,4 \\
& Alto & 13 & 35,1 \\
& Total & 37 & 100,0 \\
\hline
\end{tabular}

\section{Figura 5}

Distribución de frecuencias de la variable aprendizaje del idioma inglés

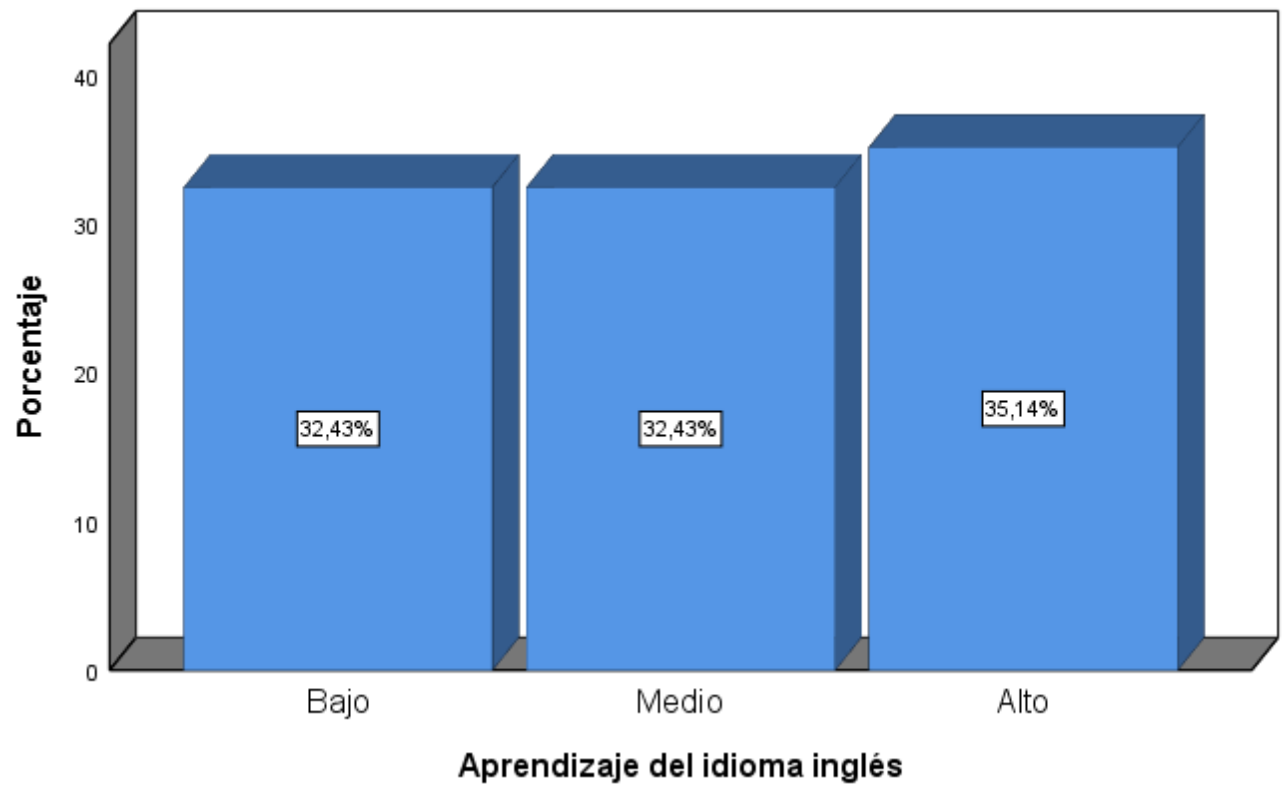

En la tabla 8 y figura 5 referente al aprendizaje del idioma inglés de los estudiantes de inglés de un instituto superior de lima, observamos que el 32.4\% (12) muestran un nivel bajo; $32.4 \%$ (12) evidencian un nivel medio; mientras que $35.1 \%$ (13) muestran un alto nivel. 
Distribución de la tabla de contingencia de las variables ansiedad de los estudiantes y el aprendizaje del idioma inglés

\section{Tabla 9}

Niveles de distribución de la tabla de contingencia entre las variables ansiedad y aprendizaje del idioma inglés como lengua extranjera de los estudiantes de educación superior

\begin{tabular}{|c|c|c|c|c|c|c|}
\hline & & & Apre & $\begin{array}{l}\text { izaje de } \\
\text { inglés }\end{array}$ & idioma & \\
\hline & & & Bajo & Medio & Alto & Total \\
\hline & Bajo & Frecuencia & 1 & 8 & 8 & 17 \\
\hline & & $\begin{array}{l}\% \text { dentro de Ansiedad de los } \\
\text { estudiantes }\end{array}$ & $5,9 \%$ & $47,1 \%$ & $47,1 \%$ & $100,0 \%$ \\
\hline Ansiedad de & Medio & Frecuencia & 10 & 3 & 5 & 18 \\
\hline $\begin{array}{c}\operatorname{los} \\
\text { estudiantes }\end{array}$ & & $\begin{array}{l}\% \text { dentro de Ansiedad de los } \\
\text { estudiantes }\end{array}$ & $55,6 \%$ & $16,7 \%$ & $27,8 \%$ & $100,0 \%$ \\
\hline & Alto & Frecuencia & 1 & 1 & 0 & 2 \\
\hline & & $\begin{array}{l}\% \text { dentro de Ansiedad de los } \\
\text { estudiantes }\end{array}$ & $50,0 \%$ & $50,0 \%$ & $0,0 \%$ & $100,0 \%$ \\
\hline Total & & Frecuencia & 12 & 12 & 13 & 37 \\
\hline & & $\begin{array}{l}\% \text { dentro de Ansiedad de los } \\
\text { estudiantes }\end{array}$ & $32,4 \%$ & $32,4 \%$ & $35,1 \%$ & $100,0 \%$ \\
\hline
\end{tabular}

En la tabla 9 se puede apreciar que el $47.1 \%$ de los estudiantes de inglés de un instituto superior de Lima que tienen un bajo nivel de ansiedad, evidencian un alto nivel de aprendizaje del idioma inglés, también el $47.1 \%$ evidencian un nivel medio y el $5.9 \%$ un nivel bajo de aprendizaje; el $27.8 \%$ que evidencian un nivel medio de ansiedad muestran un nivel alto de aprendizaje, el $16.7 \%$ de los estudiantes que presentan un nivel medio de ansiedad también muestran un nivel medio en cuanto a su nivel de aprendizaje; el 55,6\% de los estudiantes que muestran un nivel medio de ansiedad muestran un bajo nivel de aprendizaje; mientras que el 50\% de los estudiantes que tienen un alto nivel de ansiedad muestran un nivel medio de aprendizaje y el otro $50 \%$ muestra un nivel bajo.

\section{Resultados del nivel inferencial}

\section{Prueba de bondad de ajuste (normalidad).}

Según Vargas (2005) la prueba de bondad de ajuste "Tiene una aplicación fundamental cuando se trata de averiguar si la distribución empírica que resulta de cuantificar los datos de una muestra se aproxima a la distribución normal, condición que es requerida en numerosas situaciones" (p. 390).

Para realizar esta prueba, se recurre al contraste de normalidad de Shapiro -Wilk según Vargas (2005), "Es la prueba más recomendable para testar la normalidad de una muestra, con 
datos menores a 50. Se basa en medir el ajuste de los datos a una recta probabilística Normal. El estadístico de contraste se expresa por medio de la siguiente ecuación" (p. 395).

Para realizar la aplicación de la Shapiro - Wilk fue necesario utilizar el software estadístico SPSS 25 y seguir los siguientes pasos según Vargas (2005):

Paso 1. Plantear la hipótesis nula (Ho) y la hipótesis alternativa (H1):

Ho: Los datos provienen de una distribución normal

Ha: Los datos no provienen de una distribución normal

Paso 2. Seleccionar el nivel de significancia

Para efectos de la presente investigación se ha determinado que: $\alpha=0,05$

Paso 3: Escoger el valor estadístico de prueba

El valor estadístico de prueba que se ha considerado para la presente hipótesis es Shapiro - Wilk.

\section{Tabla 10}

Prueba de normalidad

\begin{tabular}{|c|c|c|c|c|c|c|}
\hline & Kolm & ov- & & & ro-V & \\
\hline & Estadístico & gl & Sig. & Estadístico & gl & Sig. \\
\hline Ansiedad &, 112 & 37 & $200^{*}$ & ,908 & 37 &, 005 \\
\hline $\begin{array}{l}\text { Aprehensión } \\
\text { comunicativa }\end{array}$ &, 117 & 37 & ,200* & ,949 & 37 & ,088 \\
\hline $\begin{array}{l}\text { Temor a la evaluación } \\
\text { negativa por parte de los } \\
\text { compañeros }\end{array}$ & ,301 & 37 &, 000 & ,627 & 37 & ,000 \\
\hline $\begin{array}{l}\text { Ansiedad frente a los } \\
\text { exámenes }\end{array}$ & , 146 & 37 &, 046 & ,916 & 37 & ,009 \\
\hline $\begin{array}{l}\text { Aprendizaje del idioma } \\
\text { inglés }\end{array}$ &, 173 & 37 &, 007 & ,896 & 37 & ,002 \\
\hline $\begin{array}{l}\text { Vocabulario y } \\
\text { gramática }\end{array}$ &, 280 & 37 &, 000 & ,868 & 37 &, 000 \\
\hline $\begin{array}{l}\text { Pronunciación y } \\
\text { entonación }\end{array}$ &, 154 & 37 &, 027 & ,926 & 37 & ,017 \\
\hline $\begin{array}{l}\text { Contenido y límite de } \\
\text { tiempo }\end{array}$ & ,230 & 37 &, 000 & ,894 & 37 & ,002 \\
\hline
\end{tabular}




\section{Paso 4: Formulación de regla de decisión \\ Si alfa (Sig) > 0,05; Se acepta la hipótesis nula \\ Si alfa $(\mathrm{Sig})<0,05 ;$ Se rechaza la hipótesis nula}

Paso 5: Toma de decisión

En la tabla 10 se observa que, el valor calculado de significancia del estadístico de prueba de normalidad resulta en algunas componentes menores al valor teórico $\alpha=0,05$; por lo tanto, se rechaza la hipótesis nula y se acepta la hipótesis alternativa. Según esto se asume que los datos de la muestra de estudio no provienen de una distribución normal. Por lo tanto, para el desarrollo de la prueba de hipótesis; se utilizará la prueba no paramétrica del Coeficiente de Spearman para distribución no normal, a un nivel de significancia de 0,05 .

\section{Prueba de hipótesis general.}

Ha: Existe influencia significativa de la ansiedad en el aprendizaje del idioma inglés como lengua extranjera en estudiantes de un instituto de educación superior de Lima.

Ho: No existe influencia significativa de la ansiedad en el aprendizaje del idioma inglés como lengua extranjera en estudiantes de un instituto de educación superior de Lima.

\section{Nivel de significancia.} de $95 \%$

El nivel de significancia teórica es de $\alpha=0,05$, que corresponde a un nivel de confiabilidad

\section{i.Función de prueba.}

La prueba de hipótesis se realizó por medio de la prueba del coeficiente de Spearman, ya que las variables no presentan normalidad en sus datos.

\section{ii.Regla de decisión.}

Rechazar Ho cuando la significancia observada " $p$ " de los coeficientes del modelo es menor que $\alpha$.

No rechazar Ho cuando la significancia observada " $p$ ” de los coeficientes del modelo es mayor que $\alpha$. 


\section{Tabla 11}

Prueba de correlación del coeficiente rho de spearman entre la ansiedad y el aprendizaje de inglés de los estudiantes

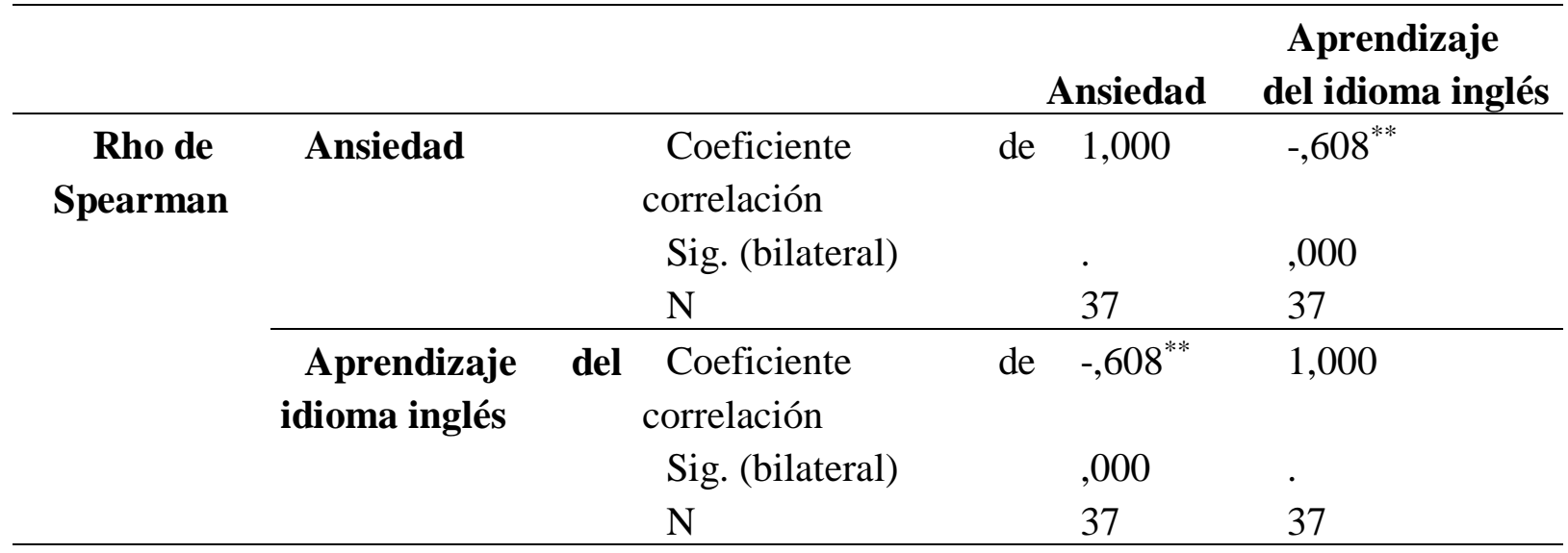

**. La correlación es significativa en el nivel 0,01 (bilateral).

\section{Grado de relación entre las variables}

En la tabla 11 se observa según los resultados del coeficiente de correlación Rho de Spearman la existencia de una relación rs $=-, 608 * *$ entre las variables ansiedad y aprendizaje del idioma inglés indicándonos que existe una relación negativa y significativa, es decir que cuando el nivel de ansiedad es bajo, el nivel de aprendizaje es alto.

\section{Decisión estadística.}

Apreciamos que el valor de significancia observada del coeficiente de Rho de Spearman $\mathrm{p}=, 000$ es menor al valor de la significancia teórica $\alpha=0,05$, lo que permite señalar que la relación es estadísticamente significativa, por lo tanto, se rechaza la hipótesis nula. Es decir, existe relación significativa entre las variables ansiedad y aprendizaje del idioma inglés de los estudiantes de un instituto superior de Lima.

\section{Discusión}

El presente estudio se ha centrado en la presencia de la ansiedad en el aprendizaje de lenguas extranjeras. La contribución más relevante es evidenciar el nivel de ansiedad de 37 jóvenes con estudios en educación superior que aprenden inglés en su institución como lengua extranjera. Además, esta investigación muestra los diferentes niveles de ansiedad en lo que respecta a sus tres componentes: la aprensión comunicativa, el temor a la evaluación negativa por parte de los compañeros y la ansiedad frente a los exámenes.

Los resultados muestran que la mayoría de los estudiantes tienen un nivel de ansiedad medio lo que coincide con otros estudios como el de Ortega Cebreros (2003) y Arnaiz y Guillen 
Influencia de la ansiedad en el aprendizaje del idioma inglés como lengua extranjera en estudiantes de educación superior

(2012) donde los participantes de las respectivas investigaciones son estudiantes universitarios. Además, coincide con el estudio de Cuétara (2015) donde los participantes son estudiantes de primaria obteniendo el mismo resultado.

El componente donde los estudiantes experimentan un nivel de ansiedad alto es la ansiedad frente a los exámenes. Esto coincide con un estudio realizado por Yang (2017) donde revela que, si un estudiante no cuenta con las habilidades comunicativas suficientes para expresarse de manera óptima, su nivel de ansiedad se incrementa frente a la realización del examen. Del mismo modo Turriate (2018) indica que este componente incide significativamente en el estudiante al permitirle o no lograr las competencias necesarias en la segunda lengua.

En segundo lugar, tenemos al componente temor a la evaluación negativa por parte de los compañeros que coincide con un estudio de Duffield (2018) en el que los resultados muestran que los estudiantes tienen temor de cometer errores al momento de comunicarse en la lengua extranjera y de ese modo quedar en ridículo delante de sus compañeros generando comparaciones entre ellos mismos.

En tercer lugar, para el componente aprensión comunicativa los resultados muestran que no existe un nivel de ansiedad elevado lo que difiere de estudios como el de Aida (1994), Pérez Paredes y Martínez Sánchez (2000- 2001) y Arnaiz y Guillen (2012) donde este componente ocupa el primer lugar entre los estudiantes.

Finalmente, en lo que se refiere a la relación de la ansiedad y el aprendizaje del idioma inglés, en este caso lo concerniente a la habilidad speaking, los resultados muestran que los estudiantes con un bajo nivel de ansiedad tienen un alto nivel de aprendizaje mientras los que tienen un alto nivel de ansiedad tienen un nivel medio y bajo en cuanto a dicho aprendizaje. Además, los resultados evidencian que los componentes donde los estudiantes tienen más dificultades son: vocabulario y gramática seguida de la pronunciación y entonación. Estos resultados difieren de un estudio realizado por Carrera y Saulo (2015) en estudiantes universitarios que pertenecían al grupo de intervención donde se evidenció un uso adecuado del vocabulario además de la correcta pronunciación, fluidez y entonación.

\section{Conclusiones}

Este estudio concluye que la mayoría de estudiantes experimenta un nivel de ansiedad medio respondiendo a la pregunta de investigación sobre cuál es el nivel de ansiedad que experimentan los estudiantes de un instituto de educación superior de Lima ante el aprendizaje de inglés como lengua extranjera.

La principal causa de ansiedad entre los estudiantes son los exámenes ya que el resultado de los mismos tiene repercusión en su futuro influyendo negativamente en el rendimiento del estudiante debido a que para algunos genera sentimientos de frustración. La segunda fuente principal de ansiedad es el temor a la evaluación negativa por parte de los compañeros debido a que los estudiantes se encuentran en constante comparación en lo referente a su desenvolvimiento durante la clase. La última causa de ansiedad es la aprehensión comunicativa relacionada a la timidez y al no comunicarse de manera apropiada en la segunda lengua. 
Como se aprecia, la ansiedad se presenta a través de estos tres componentes durante el proceso de aprendizaje de una lengua extranjera influyendo de manera negativa y afectando el desenvolvimiento de los estudiantes. Por esta razón, es vital identificar las manifestaciones de ansiedad que muestran de diversas maneras los estudiantes para poder ayudarlos a sobrellevar dicha situación oportunamente y no verse perjudicados.

Después de la valoración y discusión de los resultados se concluye que sí hay correlación entre las variables. Esta es una relación inversa, es decir, a mayor nivel de ansiedad el nivel de aprendizaje de la lengua extranjera es menor interfiriendo negativamente en dicho proceso de los estudiantes y ratificando nuestra hipótesis general: si existe influencia significativa de la ansiedad en el aprendizaje del idioma inglés como lengua extranjera en estudiantes de un instituto de educación superior de Lima.

En este contexto, es importante recalcar que las autoridades educativas tengan en cuenta este tipo de investigaciones para que los docentes trabajen en base a ellas y así mejorar el proceso de aprendizaje. El objetivo es disminuir la ansiedad de los estudiantes al momento de dominar de manera apropiada una lengua extranjera, en este caso el inglés, utilizando estrategias y herramientas adecuadas que nos faciliten el acercamiento con nuestros estudiantes. De esta manera no se sentirían tan presionados y nosotros, los docentes, obtendríamos mayor confianza y probablemente se notaría un cambio a mejor en cuanto a las evaluaciones.

\section{Referencias bibliográficas}

Aida, Y. (1994). Examination of Horwitz, Horwitz, and Cope's Construct of Foreign Language Anxiety: The Case of Students of Japanese. The Modern Language Journal, 78(2), 155-168. https://doi.org/10.2307/329005

Alcántara, D. F. (1992): «La ansiedad en la adquisición de la L2: Su expresión por medio de la escala F.L.C.A.S.» en Etxeberria, f. y Arzamendi, J. (eds.): Bilingüismo y Adquisición de Lenguas. Actas del IX Congreso Nacional de AESLA, Bilbao, Servicio editorial Universidad del País Vasco, 109-119.

Arnaiz, P. y Guillén, F. (2012). La ansiedad en el aprendizaje de una lengua extranjera en contexto universitario: diferencias interpersonales. Revista de Psicodidáctica. 17(1), 5-26 https://bit.ly/3zABsDt

Asociación Psiquiátrica Americana (2015) Manual de diagnóstico y estadístico de los trastornos mentales [Archivo PDF]. https://bit.ly/2WCEQPk

Bekleyen, N. (2004). Foreign language anxiety. Çukurova Üniversitesi Sosyal Bilimler Enstitüsü Dergisi, 13 (2), 27 - 40. https://kutuphane.dogus.edu.tr/mvt/pdf.php

Bertoglia, L. (2005). La ansiedad y su relación con el aprendizaje. Psicoperspectivas, 4,13 - 18. https://www.psicoperspectivas.cl/index.php/psicoperspectivas/article/viewFile/18/18

Cano, A. (1997) La visión del psicólogo. Alteraciones de los procesos cognitivos por el estrés. Medicina y Seguridad del Trabajo, XLIV, 103-111. https://bit.ly/2V0Nerz

Carrera, G. y Saulo, J. (2015) Las destrezas productivas en idiomas inglés de estudiantes universitarios. Revista Electrónica Formación y Calidad Educativa, 3. 89-109. http://refcale.uleam.edu.ec/index.php/refcale/article/view/431

Criado, R. \& Mengual, Y. (2017). Anxiety and EFL speaking in Spanish compulsory and noncompulsory secondary education: A mixed-method study. A Journal of English and 
Influencia de la ansiedad en el aprendizaje del idioma inglés como lengua extranjera en estudiantes de educación superior

American $\quad$ Studies, $\quad 55, \quad 13-35$.

https://miscelaneajournal.net/index.php/misc/article/view/515/239

Cuétara, R. (2015). Niveles de ansiedad ante el aprendizaje del inglés como lengua extranjera $(L$ E): estudio de un caso. [Tesis de maestría, Universidad de Cantabria]. https://bit.ly/3zAPTaw

Duffield, C. (2018). Ansiedad de los estudiantes españoles ante las pruebas orales de inglés como lengua extranjera. [Tesis de maestría, Universidad De Les Illes Balears]. https://bit.ly/3gJErBS

Govea, L. y Sánchez, F. (2006). La lengua extranjera y la interacción verbal en el aula. Revista de Educación Laurus, 12 (22), 208-223. https://www.redalyc.org/pdf/761/76102211.pdf

Guanipa, M. (2001). Propuesta de un Club de Conversación para los Estudiantes de Inglés como Lengua Extranjera a nivel Universitario. Trabajo Licenciatura no publicado, UNEFM, Santa Ana de Coro.

Hernández R., Fernández C. y Baptista P. (2004) Metodología de la Investigación. México, Ed. McGraw- Hill.

Horwitz, E. Horwitz, M. \& Cope, J. (1986). Foreign Language Classroom Anxiety. The Modern Language Journal, 70 (2), 125-132. https://doi.org/10.2307/327317

Horwitz, E. (2001). Language Anxiety and Achievement. Annual Review of Applied Linguistics, 21, 112- 127. https://www.cambridge.org/core/journals/annual-review-of-appliedlinguistics/article/abs/language-anxiety-andachievement/4DBB97FCB69BD1632CBBDAD96C81884E

Krashen, S. (1987). Principles and practice in second language acquisition. United Kingdom, Ed. Oxford.

Lineamientos académicos generales (2018). Recuperado de http://www.minedu.gob.pe/ley-deinstitutos/pdf/lineamientos-academicos-generales-2018.PDF

MacIntyre, P. (1999). Language anxiety: A review of the literature for language teachers. Affect in foreign language and second language learning: A practical guide to creating a lowanxiety classroom atmosphere. Boston, Ed. McGraw-Hill.

MacIntyre, P. \& Gardner, R. (1991). Language anxiety: Its relationship to other anxieties and to processing in native and second languages. Language Learning, 41(4), 513-534. https://doi.org/10.1111/j.1467-1770.1991.tb00691.x

MacIntyre, P. \& Gardner, R. (1994). The subtle effects of language anxiety on cognitive processing in the second language. Language Learning, 44 (2), 283-305. http://faculty.capebretonu.ca/pmacintyre/research_pages/journals/subtle_anxiety1994.pdf

Ortega Cebreros, A. M. (2003). Measuring Language Anxiety perceived by Spanish University students of English. BELLS: Barcelona English Language and Literature Studies, 12, 5062.

Pérez-Paredes, P. y Martínez- Sánchez, F. (2000-2001). A spanish version of the Foreign classroom anxiety Scale: revisiting Aida's factor analysis. Revista Española de lingüística aplicada, $14, \quad 227-352$. https://www.researchgate.net/publication/260124496_A_spanish_version_of_the_foreing _language_classroom_anxiety_scale_revisiting_aidas_factor_analysis

Sierra, J. Ortega, V. y Zubeidat, I. (2003) Ansiedad, angustia y estrés: tres conceptos a diferenciar. Revista Mal-estar E Subjetividade 3 (1), 10-59. www.redalyc.org/pdf/271/27130102.pdf

Statista (18 de marzo del 2021). Los idiomas más hablados en el mundo a fecha de 2020 https://es.statista.com/estadisticas/635631/los-idiomas-mas-hablados-en-el-mundo/ 
Turriate, J. (2018) Relación entre el nivel de ansiedad ante los exámenes y el logro de competencias en inglés en estudiantes del centro de idiomas UCV, Chimbote-2018. [Tesis de maestría, Universidad Tecnológica del Perú]. http://repositorio.utp.edu.pe/handle/UTP/1855

Virues, R. (2005) Estudio sobre ansiedad. Revista Psicología Científica. Com, 7(8) https://www.psicologiacientifica.com/ansiedad-estudio/

Yang, Y. (2017). Test anxiety analysis of Chinese college students in computer-based spoken English test. Educational Technology \& Society, 20(2), 63-73. https://pdfs.semanticscholar.org/6ef3/dbfbdedef8d80956d2b829a7d88e5311dfd1.pdf?_g $\mathrm{a}=2.226514589 .1859599927 .1615484799-618510530.1613929946$

Young, D. J. (1999). Affect in foreign language and second language learning. New York, Ed. McGraw- Hill Companies. 